\title{
Super-Keplerian Frequencies in Accretion Disks. Implications for Mass and Spin Measurements of Compact Objects from X-ray Variability Studies
}

\author{
S. A. Mao \\ Harvard-Smithsonian Center for Astrophysics, Cambridge, MA 02138; \\ samao@cfa.harvard.edu \\ Dimitrios Psaltis ${ }^{1}$ and John A. Milsom \\ Physics Department, University of Arizona, Tucson, AZ 85721
}

\begin{abstract}
The detection of fast quasi-periodic variability from accreting black holes and neutron stars has been used to constrain their masses, radii, and spins. If the observed oscillations are linear modes in the accretion disks, then bounds can be placed on the properties of the central objects by assuming that these modes are locally sub-Keplerian. If, on the other hand, the observed oscillations correspond to non-linear resonances between disk modes, then the properties of the central objects can be measured by assuming that the resonant modes are excited at the same radial annulus in the disk. In this paper, we use numerical simulations of vertically integrated, axisymmetric hydrodynamic accretion disks to provide examples of situations in which the assumptions implicit in both methods are not satisfied. We then discuss our results for the robustness of the mass and spin measurements of compact objects from variability studies.
\end{abstract}

Subject headings: accretion disks, black hole physics, hydrodynamics

\section{INTRODUCTION}

X-ray variability studies of accreting neutron stars and black holes offer a tool for constraining the masses and spins of the compact objects themselves. In the least modeldependent argument, the radial extent of the region responsible for variability observed with

\footnotetext{
${ }^{1}$ also, Astronomy Department, University of Arizona
} 
a given characteristic frequency is assumed to be at most equal to the size of a Keplerian orbit with the same orbital frequency. Additionally, in order for this region to be able to sustain long-lived oscillatory modes, it must be larger than the radius of the innermost stable circular orbit around the compact object. This requirement then places an upper bound on the mass of the object and a lower bound on its spin (see, e.g., Kluzniak, Michelson, \& Wagoner 1990; Mushotzky, Done, \& Pounds 1993; Miller, Lamb, \& Psaltis 1998; Strohmayer 2001; for a discussion see Psaltis 2005).

The assumption implicit in the above argument is that the Keplerian frequency corresponds to the fastest dynamical timescale at any radius in the accretion disk. This is usually justified by the fact that gravity is the strongest force in the system (see, however, Alpar \& Psaltis 2008). Indeed, the study of normal modes in a viscous accretion disk shows that the frequency of the lowest order, linear, hydrodynamic mode excited at any radius has to be smaller than the local Keplerian frequency (Kato 2001).

Observations of pairs of quasi-periodic oscillations from black holes with frequencies in small-integer ratios, however, have challenged the notion that these oscillations correspond to linear hydrodynamic modes (Strohmayer 2001; Abramowicz \& Kluzniak 2001). Such frequency ratios are reminiscent of non-linear interactions, which can preferentially amplify only those high-order harmonics of the normal modes that are in resonance. If this is the prevailing mechanism for compact-object variability, then meaningful bounds on or measurement of their masses and spins can only be obtained within the context of a detailed model for the oscillations. Indeed, assuming that the two modes in resonance are dynamical hydrodynamic modes in the disk and using an independent dynamical measurement of the mass of the black hole may lead to an estimate of the black-hole spin (Abramowicz \& Kluzniak 2001; Abramowicz et al. 2003). The assumption implicit in this last argument is the fact that both modes are excited and trapped in the same radial annulus in the accretion disk.

In this paper, we use numerical simulations of axisymmetric viscous accretion disks in order to assess the validity of the two main assumptions discussed above: that the highest frequency of oscillation of a radial annulus in an accretion disk is the local Keplerian frequency and that interactions may occur only between modes that are locally excited. We show that neither of these two assumptions are formally justified.

We find that oscillatory modes that are excited close to the innermost stable circular orbit generate traveling sound waves that propagate radially to tens of Schwarzschild radii. The presence of such modes has been predicted in analytical studies of diskoseismic modes (Kato 2001). Our study shows, however, that they can grow to such amplitudes that they can dominate the power spectrum of variability of the accretion disks over extended regions. As viewed from any one radial annulus outside their excitation region, these oscillations are 
highly super-Keplerian. Moreover, non-linear couplings may lead to resonance between the traveling modes and those that are excited locally. Both results have important implications for the phenomenological constraints imposed on the masses and spins of compact objects by the observation of high-frequency quasi-periodic oscillations, which we will discuss in detail below.

\section{ONE DIMENSIONAL MODELS OF HYDRODYNAMIC ACCRETION DISKS}

We used the numerical algorithm described in Milsom \& Taam (1996) in order to solve the hydrodynamic equations that describe the time evolution of one-dimensional viscous accretion disks. For completeness, we summarize here briefly the relevant equations and assumptions.

The conservation of mass is described by the continuity equation

$$
\frac{\partial \Sigma}{\partial t}+\frac{\partial}{r \partial r}\left(r \Sigma u_{r}\right)=0
$$

where $\Sigma$ is the column density through the entire thickness of the accretion disk at radius $r$ and $u_{r}$ is the radial velocity. The conservation of linear and angular momentum are described, respectively, by the equations

$$
\frac{\partial\left(\Sigma u_{r}\right)}{\partial t}+\frac{\partial}{r \partial r}\left(r \Sigma u_{r}^{2}\right)=-\frac{\partial P}{\partial r}+\Sigma\left(\Omega^{2}-\Omega_{\mathrm{K}}^{2}\right) r
$$

and

$$
\frac{\partial\left(\Sigma \Omega r^{2}\right)}{\partial t}+\frac{\partial}{r \partial r}\left(r \Sigma u_{r} \Omega r^{2}\right)=\frac{1}{r} \frac{\partial}{\partial r}\left(\Sigma \nu r^{3} \frac{\partial \Omega}{\partial r}\right)
$$

where $P$ is the sum of the vertically integrated gas and radiation pressures, $\Omega$ is the angular velocity in the flow, $\Omega_{\mathrm{K}}$ is the local azimuthal (Keplerian) dynamical frequency, and $\nu$ is the kinematic viscosity. Finally, the conservation of energy is described by the equation

$$
\frac{\partial\left(\Sigma \epsilon_{\mathrm{I}}\right)}{\partial t}+\frac{\partial}{r \partial r}\left[r u_{r}\left(\Sigma \epsilon_{\mathrm{I}}+P\right)\right]=u_{r} \frac{\partial P}{\partial r}+\nu \Sigma\left(r \frac{\partial \Omega}{\partial r}\right)^{2}-\frac{4 a c T^{4}}{3 \kappa_{0} \Sigma}
$$

where $\epsilon_{\mathrm{I}}$ is the internal energy per unit mass, $T$ is the mid-plane temperature in the disk, $a$ is the radiation constant, $c$ is the speed of light, and $\kappa_{0}$ is the sum of the electron-scattering and free-free opacities of the flow.

This set of differential equations is augmented by a set of algebraic equations that determine the gravitational potential of the central star, the equation of state, and the 
kinematic viscosity in the flow. We specify a pseudo-Newtonian gravitational potential through

$$
\Omega_{\mathrm{K}}=\sqrt{\frac{G M}{r\left(r-r_{\mathrm{S}}\right)^{2}}},
$$

where $r_{\mathrm{S}} \equiv 2 G M / c^{2}$ is the Schwarzchild radius that corresponds to the mass of the star, $M$. We use an equation of state that corresponds to a perfect gas in local thermodynamic equilibrium with radiation such that

$$
\epsilon_{\mathrm{I}}=\frac{3}{2} \frac{\mathcal{R} T}{\mu}+\frac{2 H a T^{4}}{\Sigma}
$$

and

$$
P=P_{\text {gas }}+P_{\text {rad }}=\frac{\Sigma \mathcal{R} T}{\mu}+\frac{2}{3} H a T^{4},
$$

where $\mathcal{R}$ is the gas constant, $\mu=0.62$ is the mean molecular weight in the flow, $a$ is the radiation constant, and

$$
H \equiv \frac{\sqrt{P / \Sigma}}{\Omega_{\mathrm{K}}}
$$

is the vertical scale height of a disk in hydrostatic equilibrium. Finally, we use the $\alpha$ prescription

$$
\nu=\frac{2}{3} \alpha \frac{P_{\mathrm{gas}}}{P} \sqrt{\frac{P}{\Sigma}} H
$$

for the kinematic viscosity, which ensures thermal and viscous stability.

In all numerical simulations we set the mass of the central object to $10 M_{\odot}$ and specified the solution by the mass accretion rate $\dot{M}$ in units of the Eddington accretion rate $\dot{M}_{\mathrm{E}} \equiv$ $64 \pi G M /\left(k_{\mathrm{es}} c\right)$ and the viscosity parameter $\alpha$. The domain of solution, initial conditions, boundary conditions, and method of solution are described in detail in Milsom \& Taam (1996).

The global timing properties of our numerical simulations have been described in detail by Milsom \& Taam (1996). In summary, for a given value of the viscosity parameter $\alpha$, simulations with low (typically $\lesssim 0.01 \dot{M}_{\mathrm{E}}$ ) accretion rates result in lightcurves with noisy power spectra, simulations with intermediate accretion rates exhibit large amplitude global modes at frequencies comparable to the maximum radial epicyclic frequency in the flow and its harmonics, and simulations at even larger accretion rates (typically $\gtrsim 0.02 \dot{M}_{\mathrm{E}}$ ) exhibit no significant variability (although epicyclic oscillations were always visible in subsequent 2D hydrodynamic simulations; see Milsom \& Taam 1997). 


\section{SUPER-KEPLERIAN FREQUENCIES IN THE NUMERICAL SIMULATIONS}

The main goal of our study is to investigate the radial propagation of the traveling modes excited in the various simulations, in order to quantify the extent of the domain in which they dominate the variability. For this reason, we performed Fast Fourier Transforms (FFTs) of a number of physical quantities such as the radiation flux, local mass accretion rate, vertically integrated column density, etc., evaluated at different radii in the flow. In order to resolve the fastest possible oscillations we used a time resolution of $2 \mathrm{~ms}$, and in order to resolve the power-spectral peaks of potential oscillations of high coherence we used segments of $4 \mathrm{~s}$ length. Finally, following standard procedure, we averaged the FFTs of four sequential segments in every simulation, in order to improve the statistics, and binned the power density spectra in frequency space. Hereafter, we will be discussing the power density spectra of the vertically integrated column density in the disk.

Figure 1 shows a typical example of the power spectrum of the vertically integrated density $\Sigma$ evaluated at three different radii in the accretion disk for the simulation with an accretion rate of $0.1 \dot{M}_{\mathrm{E}}$ and a viscosity parameter of $\alpha=0.1$. We can clearly see that the accretion disk is variable over a wide range of timescales and, similar to the observed systems, exhibits both broad-band noise and quasi-periodic oscillations. Indeed, at $6 R_{\mathrm{S}}$ a large number of oscillation peaks can be seen, most of which are overtones to a fundamental frequency. However, contrary to all expectations, most of the quasi-periodic oscillations that appear in the power spectra occur at frequencies larger than the local Keplerian frequencies, indicated in the Figure with the vertical dashed lines.

Figure 2 shows the frequency of the dominant quasi-periodic oscillation of the vertically integrated density as a function of radius, for the simulation with $\dot{M}=0.1 \dot{M}_{\mathrm{E}}$ and a viscosity parameter $\alpha=0.1$. The radius dependence of the oscillation frequency depicted in this figure shows three distinct regions that appear in varying degrees in all simulations. First, there is a wide region in the inner accretion disk where the dominant oscillation frequency is constant and nearly equal to the maximum of the radial epicyclic frequency. This is surrounded by a second region in which the oscillation frequency is radius-dependent, rapidly decreasing, but super-Keplerian. Finally, in a third, outer region, the oscillation frequency is radiusdependent and nearly equal to the radial epicyclic (or local Keplerian) frequency.

The radial extent of the regions in the accretion disk in which the constant-frequency and variable-frequency modes live depends strongly on the mass accretion rate and rather weakly on the viscosity parameter $\alpha$, as shown in Figure 3. At accretion rates smaller than a few hundredths of the Eddington critical value, the oscillation frequencies at all radii are nearly equal to the local epicyclic frequencies. As the accretion rate increases beyond $\sim 0.01 \dot{M}_{\mathrm{E}}$, 
the radial extent of the constant-frequency mode also increases, reaching radii $\gtrsim 40 R_{\mathrm{g}}$ for accretion rates comparable to $0.1 \dot{M}_{\mathrm{E}}$; beyond that radius, only the variable frequency mode exists.

The constant frequency modes that dominate the variability in the inner accretion disk at accretion rates $\simeq 0.1 \dot{M}_{\mathrm{E}}$ are neither excited nor trapped in that region. They are in fact traveling inertial-acoustic modes that are generated close to the innermost stable circular orbit and propagate outwards (see also Chen \& Taam 1995). This is illustrated in Figure 4, which shows the radius-time plot of the vertically integrated column density for the simulation with an accretion rate of $0.1 \dot{M}_{\mathrm{E}}$ and a viscosity parameter $\alpha=0.1$.

\section{DISCUSSION}

We investigated the presence of oscillatory radial modes in numerical simulations of axisymmetric, viscous accretion disks. In agreement with earlier efforts (see Chen \& Taam 1995), we find that traveling modes are generated close to the innermost stable circular orbit of the flow and propagate outwards to several tens of Schwarzschild radii, without getting attenuated. As viewed from any given annulus in the accretion disk, these waves appear as density oscillations with frequencies that are locally super-Keplerian.

The presence of such super-Keplerian oscillations in the simulations implies that we cannot use the frequency of an observed oscillation to set a bound on the extent of the region in the accretion flow that is responsible for the modulated emission. For example, if we were to assume, as is normally done, that the oscillation with a frequency $f \simeq 110 \mathrm{~Hz}$ shown in Figure 2 is limited by the local Keplerian frequency, i.e., that $f \leq f_{\mathrm{K}} \simeq(1 / 2 \pi) \sqrt{G M / R^{3}}$, then we would infer that the region responsible for this oscillation is smaller than

$$
R \leq 4.7 R_{\mathrm{S}}\left(\frac{M}{10 M_{\odot}}\right)^{1 / 3}\left(\frac{f}{110 \mathrm{~Hz}}\right)^{-2 / 3} .
$$

However, this limit would seriously underestimate the radial extent of the region where the $\simeq 110 \mathrm{~Hz}$ mode resides, which is in fact four times larger (see Fig. 2). This is a direct consequence of the fact that the traveling mode is locally super-Keplerian in the entire inner region of the disk.

It is important to emphasize here that, although the constant-frequency modes are super-Keplerian in the region of the accretion disk where they are traveling, they are subKeplerian near the innermost stable circular orbit, where they are excited. This is true for all modes in all simulations that we explored. As a result, even though we cannot use the frequency of the mode to set an upper bound on the radial extent of the region in which it 
resides, we can still constrain the mass and the spin of the compact object by requiring that the mode is sub-Keplerian in the region where it is excited.

Our simulations can only recover the excitation and propagation of axisymmetric radial oscillations. Because of this, we cannot simulate the possible non-linear coupling of these to other, azimuthal or vertical, modes. However, the presence of large amplitude traveling modes in the simulated disks suggests that the non-linear coupling and resonances may not necessarily occur between modes that are excited in the same region in the accretion disk. Indeed, Figure 2 shows that the $\simeq 110 \mathrm{~Hz}$ mode is in 2:1 resonance with an azimuthal Keplerian mode at $\simeq 7 R_{\mathrm{S}}$, in 3:2 resonance with an azimuthal mode at $\simeq 8.5 R_{\mathrm{S}}$, etc. As a result, it is not justifiable to use the frequencies of observed resonant oscillations and infer the properties of the compact objects assuming that they correspond to modes in the same region in the accretion flow (as in, e.g., Abramowicz \& Kluzniak 2001).

Finally, it is worth pointing out that our results are valid for geometrically thin, hydrodynamic accretion disks with an alpha viscosity. Traveling inertial-acoustic modes such as those we studied here are not visible in time-dependent simulations of magnetohydrodynamic accretion disks (see, e.g., Hawley \& Krolik 2001; Armitage \& Reynolds 2003; but see also Arras, Blaes, \& Turner 2006 and Chan et al. 2008). This is probably due to the combined effects of the magnetorotational instability, which damps such radial epicyclic modes, and the presence of fully developed turbulence that quickly destroys the coherence of such traveling modes. It is not clear, at this point, what physics needs to be included (or perhaps what assumptions need to be relaxed) for the numerical simulations of magnetohydrodynamic disks

to show large-amplitude oscillations that are ubiquitous in nature. Independent of whether the hydrodynamic inertial-acoustic modes survive in realistic simulations, our results still serve as a proof of principle that high amplitude, super-Keplerian traveling modes may, in principle, exist in the inner regions of accretion flows.

D. P. was supported in part by the NSF CAREER award NSF 0746549.

\section{REFERENCES}

Abramowicz, M. A., \& Kluzniak, W. 2001, A\&A 374, L19

Abramowicz, M.A., Karas, V., Kluzniak, W., Lee, W.H., \& Rebusco, P. 2003, PASJ, 55, 467

Armitage, P. J., \& Reynolds, C. S. 2003, MNRAS, 341, 1041 
Arras, P., Blaes, O., \& Turner, N. J. 2006, ApJ, 645, L65

Alpar, M. A., \& Psaltis, D. 2008, MNRAS, submitted

Chan, C. K., et al. 2008, ApJ, submitted, astro-ph/0611269

Chen, X., \& Taam, R. E. 1995, ApJ, 441, 354

Hawley, J. F., \& Krolik, J. H. 2001, ApJ, 548, 348

Kato, S. 2001, PASJ, 53, 1

Kluzniak, W., Michelson, P., \& Wagoner R. V. 1990, ApJ, 358, 538

Miller, M. C., Lamb, F. K., \& Psaltis, D. 1998, ApJ, 508, 791

Milsom, J. A., \& Taam, R. E. 1996, MNRAS, 283, 919

—. 1997, MNRAS, 286, 358

Mushotzky, R.F., Done, C., \& Pounds, K. A. 1993, ARA\&A, 31, 717

Psaltis, D. 2004, in X-ray Timing 2003: Rossi and Beyond, 714, 29

Strohmayer, T. E. 2001, ApJ, 552, L49 


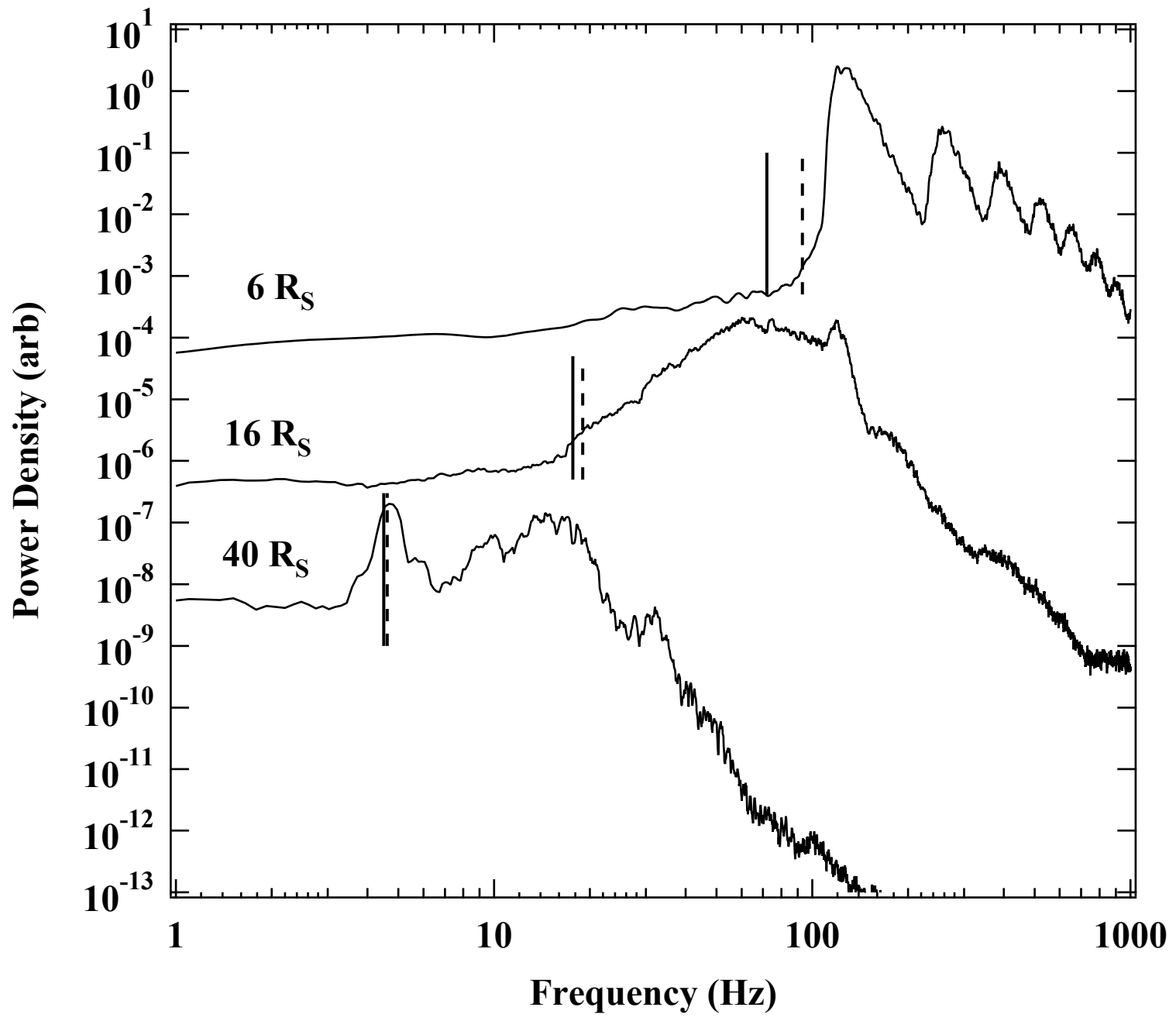

Fig. 1. - The power spectrum of the vertically integrated column density $\Sigma$ at three different radii in an accretion disk with an accretion rate of $0.1 \dot{M}_{\mathrm{E}}$ and a viscosity parameter $\alpha=0.1$. The power spectra have been displaced vertically for clarity. The vertical solid line on each power spectrum corresponds to the local radial epicyclic frequency, whereas the vertical dashed line on each power spectrum corresponds to the local Keplerian frequency. 


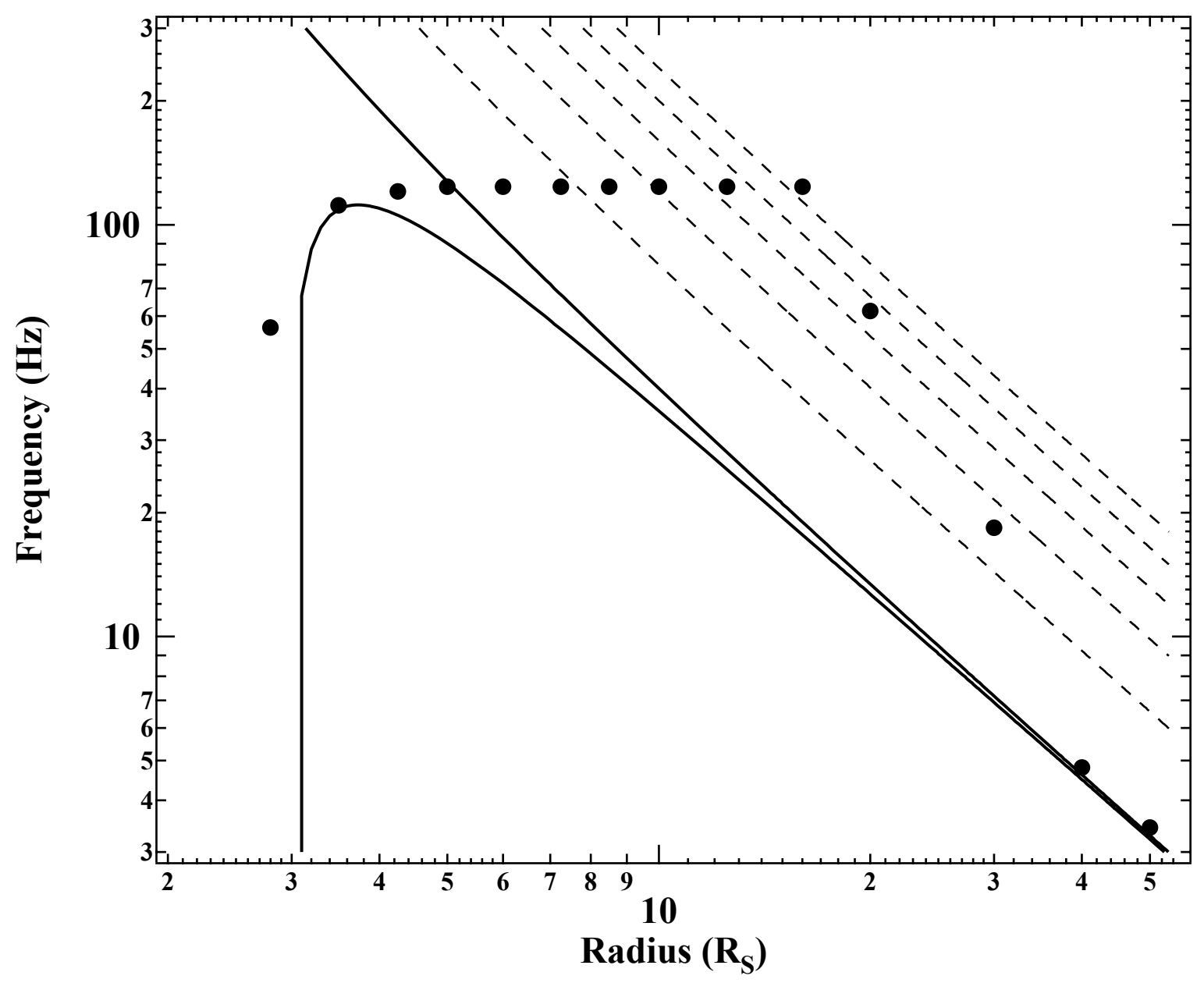

Fig. 2.- The radius dependence of the dominant oscillation frequency of the vertically integrated density in a simulation with $\dot{M}=0.1 \dot{M}_{\mathrm{E}}$ and a viscosity parameter $\alpha=0.1$. At each radius, the solid lines show the radial epicyclic and Keplerian frequencies, whereas the dashed lines show increasingly higher harmonics of the local Keplerian frequency. 


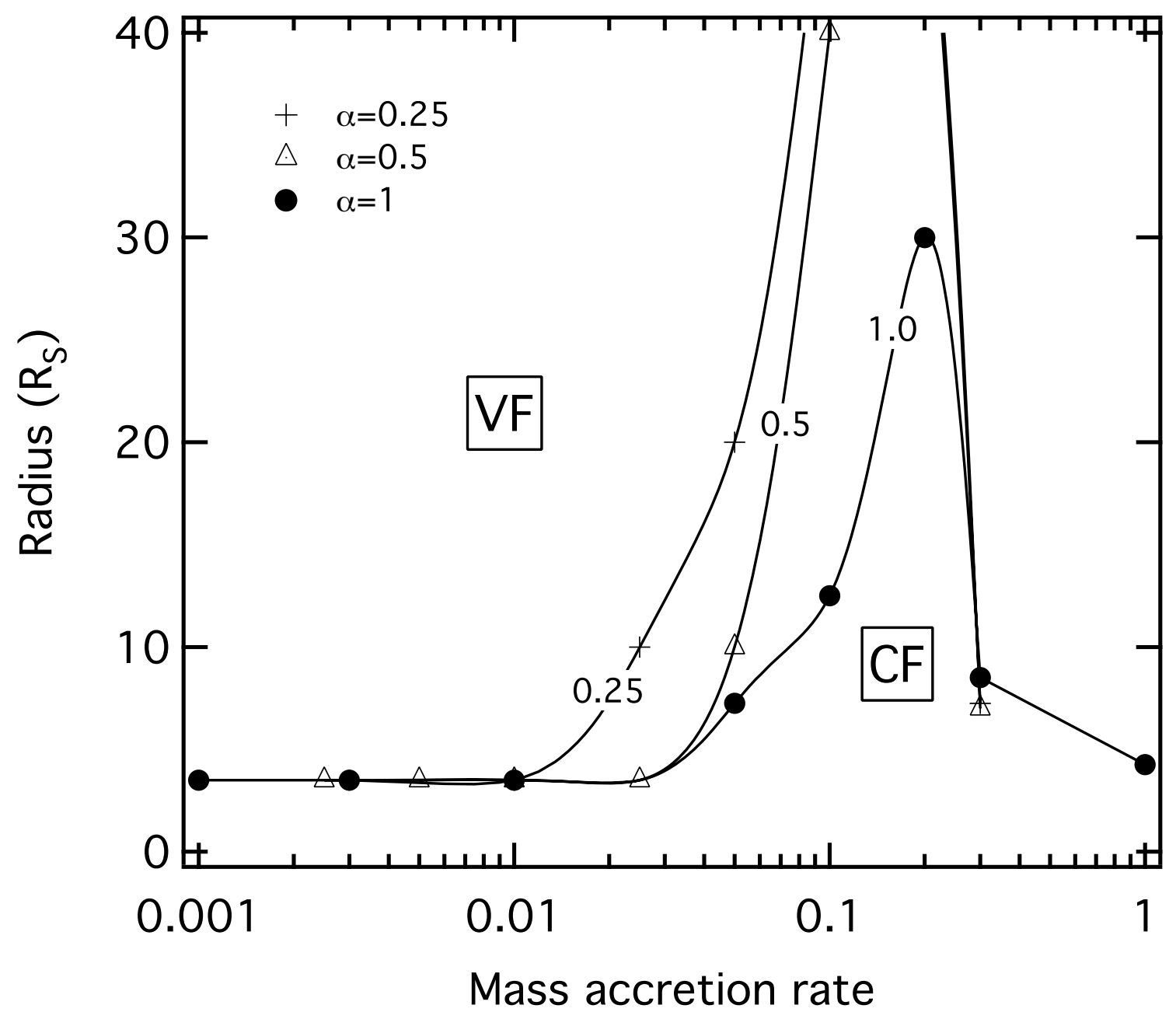

Fig. 3.- The regions in the accretion disk where the constant-frequency $(\mathrm{CF})$ and the variable-frequency (VF) modes live for simulations with different mass accretion rates and viscosity parameters $\alpha$. 


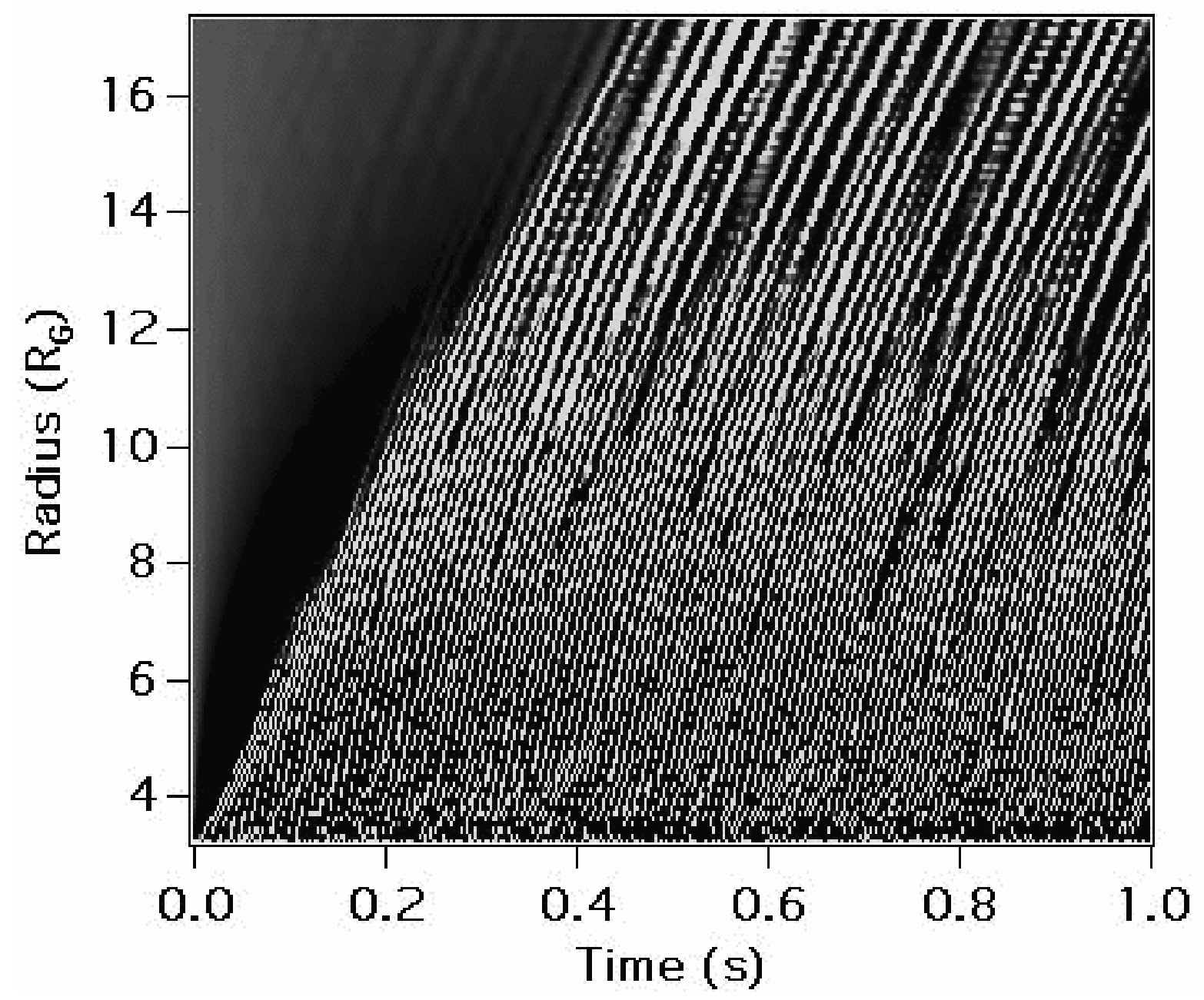

Fig. 4. - A radius-time plot of the vertically integrated density for the simulation with an accretion rate of $0.1 \dot{M}_{\mathrm{E}}$ and a viscosity parameter $\alpha=0.1$. 